\title{
APROXIMACIÓN AL PROBLEMA DE LA FORMACIÓN DEL CANON EN LITERATURA COMPARADA
}

\section{Dolores Romero López}

Grupo de Investigación del ISLTYNT (UNED)

El florecimiento actual de los estudios sobre la formación del canon literario invita a plantear una reflexión sobre el contexto histórico y teórico en que se encuentra esta investigación hoy en día. Si ya supone un desafío abordar el problema de la formación del canon en un momento determinado de una única literatura nacional, como ha puesto de manifiesto Luis González del Valle (1993); si resulta más complejo analizarlo en el terreno de la teoría de la literatura, como ha hecho José M. ${ }^{a}$ Pozuelo Yvancos (1995), lo cierto es que plantear el problema de la formación del canon en el marco teórico de la literatura comparada supone un reto intelectual que no admite parámetros restrictivos, sino una perspectiva cuestionadora y abierta. Desde el comparativismo quizá sea posible percibir con mayor nitidez cuáles son las líneas de fuerza que rigen las diversas posturas sobre el canon, así como cuáles son las cuestiones teóricas más urgentes para abordar su problemática. 
En este planteamiento ha influido una convicción propia que parte del principio de que la teoría no ha de confundirse con la reflexión desarraigada acerca de problemas intemporales. La teoría literaria ha de esforzarse por asentar en las propias circunstancias históricas y culturales el interés que despierta una determinada línea de investigación. ¿Cuál es, pues, la justificación de este planteamiento sobre el problema del canon en la España de finales del siglo XX? Por el momento dejaré esta pregunta en el aire y trataré de irla encauzando a lo largo de la presente argumentación.

Permítaseme que me inspire al comienzo de esta investigación en unas palabras que Jorge Luis Borges escribe en «La biblioteca de Babel»:

Cuando se proclamó que la Biblioteca abarcaba todos los libros, la primera impresión fue de extravagante felicidad. Todos los hombres se sintieron señores de un tesoro intacto y secreto (Borges, 1985: 94).

Esa biblioteca global e infinita, ilimitada y periódica, que describe Borges en su narración, es el abrumador objeto de estudio de la literatura comparada. Pero esa biblioteca, precisamente por ser una creación humana, está sujeta a ciertos principios vertebradores que abarcan tanto la disposición de los libros en los anaqueles como la combinación de los signos elementales que reflejan la variedad idiomática y cultural del conocimiento humano. En este sentido, para que la biblioteca funcione, necesita de un sistema de reglas que permita el desarrollo del conocimiento, o, como prefiere Lotman desde la Semiótica de la cultura, el desarrollo de la vida comunitaria.

En el momento en que surge la necesidad de ciertas prescripciones hace su aparición el canon que etimológicamente significa 'tallo', 'varita' y también 'regla', 'norma'. El canon fue primitivamente usado para catalogar aquellos «libros tenidos por la iglesia católica como auténticamente sagrados», según dicta su definición en el diccionario. Desde su primitiva utilización lo canónico, al ser aplicado a lo sagrado, fue sumando a su valor denotativo de catálogo o lista aquel derivado de la connotación que le vincula a lo modélico o perfecto.

Ernst Robert Curtius (1955), en su libro Literatura europea y Edad Media latina, ha estudiado el problema de la formación del canon a partir del siglo IV, cuando la Iglesia incorporó las escrituras sagradas 
de los judíos a las que dio el nombre de Antiguo Testamento. Después se sumarán al canon católico los textos de la patrística y la escolástica. Durante la Edad Media, con la creación de las primeras universidades europeas, fue menester establecer en la educación un corpus de textos reconocido y valioso que sirviera de marco de referencia. En la universidad se comenzó a yuxtaponer, frente a los autores canónicos cristianos, aquellos que provenían de una tradición pagana, de donde nace la creación del canon moderno, destacando, a partir de 1500 , la cultura italiana. En ella floreció junto a la poesía latina de Virgilio aquella que en lengua vulgar compusieron Dante y Petrarca. La necesidad de reinventar a los autores griegos y latinos dio lugar a la creación y difusión de las llamadas tendencias clasicistas. El canon retiene, entonces, la connotación de clásico y pasa a estar compuesto por obras y autores dignos de imitación que no se aparten de la tradición. Este clasicismo es el que heredó, a través de la escuela de Ronsard, la Francia de Luis XIV (1638-1715), que había logrado una poderosa hegemonía sobre Europa. Durante el imperialismo francés el clasicismo dejó de ser una imitación de los antiguos para convertirse en una configuración de contenido nacional, basado en el éxito del racionalismo galo. El espíritu nacional francés pasó a ser, por antonomasia, el espíritu universal del que surge, en los primeros lustros del siglo XIX, la literatura comparada. Este excursus histórico es fundamental para entender el posterior desarrollo del canon y su formación dentro de una perspectiva eurocentrista.

\section{LA REDEFINICIÓN DEL CANON EN LITERATURA COMPARADA}

La concepción del canon literario ha estado muy vinculada a la definición misma de la literatura comparada. René Wellek y Austin Warren (1989), en su ya clásico libro Teoría literaria, mencionan que el término de literatura comparada abarca al menos tres esferas de estudio. En primer lugar, la literatura oral, particularmente los temas populares y su entrada en la literatura culta. En segundo lugar, la literatura comparada se circunscribe a las relaciones entre dos o más literaturas. En tercer lugar - y esta es la acepción que aquí nos interesa-, la literatura comparada se ha identificado con el estudio de la literatura en su totalidad, con la literatura universal, con la literatura general 
o mundial. Esta última opinión, publicada por Wellek y Warren por primera vez en 1952, ha venido siendo ratificada por los principales especialistas. Así, por ejemplo, S. S. Prawer (1973) afirma que el campo léxico de la literatura comparada incluye, además, el de «literatura mundial» o Weltliteratur.

Los teóricos que investigan el ascenso de la disciplina se remontan a los primeros lustros del siglo XIX, cuando en Francia François Villemain pronuncio sus famosas conferencias (1828-1829) y en Alemania Goethe (1927) fraguó el término Weltliteratur. Este concepto sólo tiene sentido en el contexto de una Europa romántica que comenzaba a gestar los nacionalismos al tiempo que se veía la necesidad de superar las fronteras nacionales. Goethe no establece un canon de obras maestras, como después se ha venido haciendo al hablar de literatura universal. La Weltliteratur se concibe como mediación entre las literaturas para alcanzar el conocimiento, la comprensión, tolerancia y aceptación de otras literaturas. Pero es el afán de perpetuación de la mentalidad europea lo que determina la aparición del canon occidental, con el fin de salvaguardar el gran tesoro de los clásicos. De él forman parte los grandes autores - Homero, Dante, Cervantes, Shakespeare y Goethe-, cuya fama se ha extendido por todo el mundo, y los «libros importantes», «clásicos», «los mejores libros escritos en el mundo», entre los que se incluyen la Odisea, la Orestiada, la Eneida, la Divina Comedia, Fausto, Madame Bovary, La montaña mágica. Esta acepción de literatura comparada como literatura universal obedece a un deseo de europeizar la cultura que fue muy fuerte a principios del siglo $\mathrm{XX}$.

A la europeización del mundo y la difusión de un canon compuesto por textos europeos contribuyó también la crítica literaria. Si hay algo que destaca al leer los textos que componen el volumen publicado por H. Schulz y P. Rhein (1973), Comparative Literature: The early years, es que la literatura comparada comenzó siendo literatura europea comparada. Europa imponía su superioridad al resto del mundo, también y principalmente a través de su literatura, y a ello contribuyeron los estudios Fernand Baldensperger y Paul Hazard hacia los años veinte y posteriormente otros de Jean-Marie Carré, Marcel Bataillon, Paul Van Tieghem etc. René Wellek será el primero en denunciar este hecho durante su intervención en el segundo congreso de la ICLA, celebrado en Chapel Hill en 1958. Bajo el título «The Crisis of Comparative Literature», Wellek reprocha la obsoleta metodología decimonónica utilizada por Van Tieghem, Carré y 
Guyard. Al criticar el patriotismo que se muestra en los estudios de los eruditos franceses, alemanes e italianos, denuncia la acumulación de investigaciones que favorecen y alientan un espíritu europeo superior. A las opiniones de Wellek se suma el francés René Étiemble, quien en su estimulante Comparaison n'est pas raison de 1963 defendía que deberíamos seguir el ejemplo de Goethe y buscar fuera de Europa nuestros cánones de excelencia y el estímulo para esfuerzos literarios nuevos. Étiemble criticó muy duramente al eurocentrismo en los estudios comparados y en la formación del canon y acusó a la burguesía de destruir sistemáticamente las literaturas africanas, indias, amerindias, etc., lo que ha terminado — según él- escindiendo la literatura en literatura de amos y esclavos.

Estas opiniones y críticas vertidas por Wellek y por Étiemble abrieron un verdadero debate sobre el establecimiento del canon universal. Se comenzó, por fin, a cuestionar el canon y sus connotaciones de perfección, clasicismo y tradición. ¿Cómo se forma el canon de los grandes autores? ¿Por qué se concede más importancia al Quijote de Cervantes que a La Celestina de Fernando de Rojas cuando se establece un canon universal? ¿Por qué la Divina comedia de Dante y no El Cancionero de Petrarca? ¿Por qué Jane Austen y no Rosalía de Castro? ¿Por qué Unamuno como exponente de la Generación del 98 y no Joan Maragall? ¿Por qué Dostoiewsky había de parecer cada vez más relevante en la Europa del siglo XX? Ante tales preguntas parece conveniente abordar el problema del canon en los debates actuales.

\section{EL PROBLEMA DE LA FORMACIÓN DEL CANON EN LOS DEBATES ACTUALES}

Para entender el gran debate que sobre la formación del canon se está llevando a cabo con virulencia en el ámbito universitario norteamericano y más moderadamente en el entorno europeo, hay que señalar, en primer lugar, la gran apertura teórica que han experimentado los estudios comparativos. Las definiciones de Henry Remak (1961), Manfred Schmeling (1984), Claudio Guillén (1985) y Fridrun Rinner (1990) insisten en que la literatura comparada debería describir y comparar jerarquías pertenecientes a un corpus literario-cultural o al ámbito teórico. Con ello se da pie a que la formación del canon no esté 
integrada sólo por textos consagrados, pero en la práctica siguen sirviendo como referencia los modelos clásicos que el canon occidental ha ido perpetuando. Esto es lo que da lugar a la querella entre antiguos y modernos a la que Eva Kushner (1995) se refirió durante su intervención en el XIII Congreso de la Asociación Internacional de Literatura Comparada. Kushner abre una brecha en favor de los estudios culturales y postcoloniales, como estudios que certifican el triunfo de la comparación por suponer una defensa de la búsqueda de identidad de las diferentes culturas. La respuesta institucional en defensa de los estudios culturales en Norteamérica se debe a que prevalece la corrección política, que en ocasiones considera la complejidad de lo literario un asunto elitista. Los estudios culturales coinciden con amplios sectores de las humanidades que proponen combinar ideas postmodernas con el apetito de transgresión, y muy especialmente de quebrantamiento del canon occidental. Con la aceptación en los departamentos de literatura comparada de los llamados estudios culturales, el canon se democratiza; se generaliza de tal forma que cualquier autor y cualquier obra podrían formar parte de él. Un ejemplo de esto es el libro de Jo Labanyi y Helen Graham, Spanish Cultural Studies, que junto a lo literario contempla una amplia categoría de estudios que incluyen la sexualidad, la identidad nacional, el colonialismo y el postcolonialismo, las etnias, la cultura popular y sus audiencias, el cine, la ciencia, la ecología y las instituciones culturales de la España del siglo XX.

Con motivo de la ruptura de los estudios culturales con la ideología uniforme se crean cánones enfrentados. No olvidemos que la idea misma de canon se somete al trazado de una selección y de una historia, y ésta no es sólo ordenación del pasado sino, como advierte Jenaro Talens, una reconstrucción del mismo. El debate sobre el canon se fue ampliando gracias a las opiniones vertidas por destacados especialistas en literatura comparada. Frank Kermode en 1979 opinaba que el canon está ligado al comentario, a la crítica, y no a propiedades del texto en sí. Su instauración y perpetuación, según él, está vinculada al funcionamiento de las instituciones. Barbara H. Smith dirigió en 1983 un enconado ataque a la crítica tradicional norteamericana por haber impuesto, a la selección y perdurabilidad de obras literarias, valores asentados en la conservación de la ideología del poder. Más moderadas resultan las publicaciones de Ch. Altieri (1983) y G. Graff (1987 y 1990), que defienden un pluralismo crítico para erradicar el predominio de los sistemas cerrados y exclusivistas. En definitiva, el debate viene a poner de manifiesto lo que recoge W. Mignolo (1991): primeramen- 
te, la necesidad de superar el canon vocacional que pretende que el valor individual se convierta en un valor de cultura, y, en segundo lugar, la idea de que en la formación del canon interviene el regionalismo, es decir, aquellos valores locales que interesan a una cultura en un momento dado, con lo cual se cuestiona tajantemente el valor universalista que Occidente había venido concediendo a su canon.

Frente a estas consideraciones que terminan fragmentando y diversificando los valores en los que se asienta la canonicidad, han surgido recientemente en Estados Unidos voces divergentes. El influyente libro de Alan Bloom (1987), The Closing of the American Mind, critica la tendencia actual del sistema educativo norteamericano que permite que los estudiantes desconozcan la tradición literaria y amenaza con la pérdida de los valores clásicos de la cultura liberal democrática americana. Esta misma opinión es compartida por Harold Bloom (1996), cuyo libro, El canon occidental, publicado por primera vez en 1994 y convertido casi inmediatamente en un best-seller, surgió como crítica al multiculturalismo. Harold Bloom elige 26 autores que, según él, guardan unos patrones estéticos y antropológicos universales. El manifiesto de Bloom arranca de la defensa de una supremacía de la estética sobre la ideología. Según Harold Bloom, el canon no depende de la predilección institucional, sino de la fuerza estética de la obra y de la individualidad del autor. Cito aquí los autores incluidos en El canon occidental. En la edad aristocrática: Shakespeare, Dante, Chaucer, Cervantes, Montaigne, Molière, Milton, Samuel Johnson, Goethe; edad democrática: Wordsworth, Jane Austen, Walt Whitman, Emily Dickinson, Dickens, George Eliot, Tolstói, Ibsen; edad caótica: Freud, Proust, Joyce, Virginia Woolf, Kafka, Borges, Neruda, Pessoa y Beckett. El valor estético de sus obras radica en la originalidad que, según Bloom, se convierte en principio universal. Dicha originalidad no concierne a los contextos sociales, sino a la historia interior de los textos mismos, que encarnan una dialéctica basada en la ansiedad de la influencia.

El debate entre ideología y estética, que es el que rige el problema del canon en los Estados Unidos, no parece que tenga su contrapartida en Europa, donde se han difundido, en mayor medida, lo que Steven Tötösy de Zepetnek (1992 y 1998) denomina teorías sistémicas. Bajo dicho marbete se agrupan las corrientes que han desarrollado el concepto de sistema literario. Me refiero a la semiótica de la cultura de I. Lotman, la teoría empírica de la literatura de S. J. Schmidt y a la teoría del polisitema de Itamar Even-Zohar (1990). Me centraré exclusivamente en esta última, por la relevancia que concede al funcionamiento de la canonicidad. 
Un polisistema es un sistema de varios sistemas que se relacionan entre sí y parcialmente se superponen utilizando diferentes opciones, aunque funcionando como si fuera un todo cuyos miembros son independientes. El sistema del que habla Even-Zohar (1990) está asentado en una red de relaciones en la que sus miembros reciben los valores a través de sus respectivas oposiciones. Sólo desde esta definición se entiende que Even-Zohar no utilice el término «canon», como ha puesto de manifiesto Rakefet Sheffy, y en su lugar prefiera hablar de la «canonicidad» o, mejor, de la oposición «canonizado» frente a «no canonizado». Esta oposición justifica que los sistemas están jerarquizados y sus relaciones tienen un carácter conflictivo. Even-Zohar muestra que en el interior de un polisistema los géneros o autores no canónicos dependen de los establecidos, con los cuales rivalizan. Una concepción del polisistema basada en una oposición «canonizado»/ «no canonizado» resulta sumamente práctica porque no excluye ningún libro ni ningún autor, sino que todos los libros y todos los autores nacionales o internacionales estarían representados; su valor vendría dado no por el sujeto que lo selecciona, sino por su funcionamiento dentro del sistema en que se sitúa.

Evidentemente la teoría del polisistema viene a aportar algunas soluciones a la diatriba norteamericana, pero en la práctica pedagógica, donde el canon tiene sus verdaderas raíces, la teoría del polisistema no ofrece unas soluciones tan alentadoras como se ha querido ver. ¿Qué hacer cuando en una clase sobre las literaturas comparadas tengamos que establecer un canon de lectura? Arrimándonos más a nuestro ámbito, ¿cómo es posible establecer un acuerdo para que se estudien determinados autores y textos específicos dentro de una misma literatura nacional, pero con diferentes lenguas y culturas regionales, como es el caso de la literatura hispánica? Éste es el verdadero debate que se plantean las Humanidades en la España actual y al que la teoría del polisistema, como muy bien apunta Gerald Gillespie (1992), ofrece una posible metodología para la comprensión de la heterogeneidad lingüística y geográfica española.

Es relativamente sencillo, desde un polisistema, la explicación del funcionamiento de lo canónico, pero ¿cómo se puede establecer un canon? En un país como España existen diferentes grupos que pueden intervenir en las líneas oficiales, creando un mapa de interacciones entre ámbitos multilinguísticos y multiculturales con el tradicional poder político y lingüístico ejercido por una de ellas, en este caso el centralismo castellano. El concepto de literatura nacional española, al 
que conduce todo intento de formulación historiográfica, comienza a cuartearse ante el desarrollo y revalorización de otras literaturas regionales. El libro editado por José María Enguita y José-Carlos Mainer (1994) en torno a Literaturas regionales en España es un intento de encaminar los estudios literarios hacia el canon roto y los regionalismos. Mainer (1994: 23) dice en uno de los capítulos de ese libro, titulado «La invención de la literatura española», que, cuando hablamos de literatura española:

[N]o enunciamos un hecho natural, espontáneo e inmutable, sino un complejo hecho de cultura en el que cada uno de los dos elementos del sintagma el sustantivo y el adjetivo gentilicio han ido modificando y conformando su actual contenido.

Mainer indaga la invención de tal concepto para embestir a quienes no legitiman otra aproximación a lo literario que no sea la histórica. Detrás de ese epígrafe, «La invención de la literatura española», que tanto recuerda a aquél de Ricardo Gullón, «La invención del 98», late un intento de replantear los cánones sobre los que se ha asentado la enseñanza de la literatura española para adecuarlos a tiempos más plurales. En el fondo, la gran pregunta que surge a estas alturas de mi argumentación es ¿en qué medida es excéntrico o representativo el juicio de valor de quien selecciona un canon literario, sea éste institucional o personal?

\section{EL CANON Y EL VALOR (INTER)SUBJETIVO}

Este problema sobre el concepto de valor en la elaboración del canon ha sido analizado por dos destacados especialistas, Yves Chevrel (1980) y Douwe Fokkema (1995). Ambos autores establecen en el concepto de valor la verdadera dificultad que hoy en día entraña la formación de un canon. El valor que rige cualquier lista de autores está determinado por los procesos mediante los cuales un lector se ve conminado a clasificar una obra tomando como referencia un sistema de valores jerarquizados.

Si las obras que forman un canon tienden a perpetuarse, las preguntas que surgen son: «¿qué es lo que hace que los lectores crean que deter- 
minadas obras literarias poseen un valor duradero?», «qué es lo que hace que ciertos textos tengan una mayor posibilidad de sobrevivir que otros?» Obviamente, tanto Chevrel como Fokkema apuntan a la Rezeptionästhetik. Sin embargo, y aún teniendo en cuenta que el concepto de «horizonte de expectativas» permite describir las distintas concreciones de una obra a lo largo de su historia, su perduración en el canon no es tan simple porque los valores que gestan la formación de un canon son contingentes, relativos y plurales. Por ello no podemos analizar un proceso de evaluación literaria con independencia de nuestra propia formación como lectores y sin percibir que un texto permite varias lecturas.

A lo que ahora apunto es al constante cuestionamiento de los valores a los que recurrimos para seleccionar un corpus de libros. La singularidad de los lectores y su derecho a realizar una lectura diferente, que se atenga a su propio sistema de valores, nos lleva a cuestionar la pretensión de que una selección de textos sea mejor que otra. El corpus de textos sobre el que se trabaja en literatura comparada es, al menos teóricamente, múltiple y está en constante re-elaboración. Los valores que sirven para la creación de un canon pueden ser debatidos en cualquier momento, de forma que la elección de una obra pueda ser justificada para evitar la arbitrariedad. Cualquier obra podría formar parte del canon con tal de que se acoja al principio de dialéctica con las demás y se expongan sus valores intertextuales. Todas estas características que, según Yves Chevrel, ha de tener el corpus de textos seleccionado hacen que la disciplina de Literatura comparada sea una disciplina de riesgo.

No podemos, después de haber hecho estas consideraciones, seguir imaginándonos el canon como una criatura ideal, como un unicornio -usando el acertado símil de Gerald Gillespie-. En su lugar hay que ubicar la torpe realidad del rinoceronte, es decir, el contexto al completo. Por supuesto que seguirá existiendo una tendencia a crear centros y periferias, textos canonizados y no canonizados. Eso es inevitable. Lo que está en manos de los profesionales de la literatura comparada es establecer vínculos entre los culturas, las razas, los textos. Esas conexiones se justifican por la voluntad dialógica en la que Bajtín asienta la conciencia humana, una conciencia que nace del intercambio de un sujeto con otro. Los cánones postmodernos parecen estar asentados en la terceridad cultural; son inestables y abiertos, relativos y plurales, dialógicos y dialécticos.

Así lo entiende también, ya para terminar, Douwe Fokkema en unas palabras que hago mías. Nótese que Fokkema (1998a: 248), al hablar del canon, no alude a él de forma abstracta sino que lo personaliza: 


\begin{abstract}
Mi canon preferido, - dice él-estará guiado por la posibilidad de cambiar de código (lo contrario de la política de identidad) y pone el acento en valores contradictorios, en las diferencias entre tradiciones, en la crítica de las ideologías dominantes, y en la diversidad de modelos de conducta moral y de vida privada. Incluirá textos literarios complejos de todas las principales culturas del mundo, no sólo textos contemporáneos sino también antiguos, en la creencia de que es más gratificante intentar entender textos dificiles que suponer que se entienden otros más sencillos. Sin embargo, mis métodos de descontextualización y, con seguridad, mi atribución de significación presentista (recontex-tualización) serán diferentes de los de colegas mios, y, por tanto, mi canon preferido será diferente del suyo. Y termina diciendo Fokkema: En consecuencia, es inútil dar una relación de los textos que prefiero, puesto que cualquier lista final adoptada por un departamento de literatura comparada será siempre el resultado de un acuerdo, dictado por las diversas convicciones, por la cultura concreta en la que estemos viviendo, y por las conveniencias del momento (como la disponibilidad de los libros).
\end{abstract}

Considero que estas palabras también harían feliz a Jorge Luis Borges, cuya vasta biblioteca, ese tesoro intacto, resultaría inútil si no pudieramos desvelar sus secretos leyendo aquellos libros que nos ayuden a comprender mejor nuestra identidad.

\title{
Referencias bibliográficas
}

AltiERI, Ch. (1983). «An Idea and Ideal of a Literary Canon». Critical Inquiry 10, 37-60.

BALDENSPERger, F. (1904). Goethe en France. París: Hachette.

BALDENSPERGER, F. \& WERNER, F. (comps.) (1960). Bibliography of Comparative Literature. New York: Russel \& Russel.

Bloom, A. (1987). The Closing of the American Mind. Nueva York: Harcourt Brace.

BloOM, H. (1991). La angustia de las influencias. Caracas: Monte Ávila Editores.

- (1994). The Western Canon. The Books and Schools of the Ages. Nueva York: Harcourt Brace \& Company.

- (1996). El canon occidental. Barcelona: Anagrama.

BORGES, J. L. (1985). Ficciones. Madrid: Alianza Editorial.

Brooks, P. (1994). «Aesthetic and Ideology: What Happened to Poetics?». Critical Inquiry 10, 509-523.

Brunel, P.; PICHoIs, Cl. y Rousseau, A. M. (1983). Qu'est-ce que la littérature comparée. París: Armand Colin.

CHEVRel, Y. (1980). «Le problème de l'évaluation dans l'enseignement de la littérature comparée». En Actes du VIII ${ }^{\circ}$ Congrès de l'Association Interna- 
tionale de Littérature Comparée. Relations entre littératures de diverses cultures au XXe siècle. Littérature comparée et théorie littéraire, B. Köpeczi, \& G. Vajda G. (eds.), 2. ${ }^{\circ}$ vol., 2, 561-571. Stuttgart: Kunst und Wissen-Erich Bieber. Traduccido en Romero López, D. (ed.) (1998a).

CURTIUs, E. R. (1955). Literatura europea y Edad Media latina. México: FCE.

ÉTIEMBLE, R. (1963). Comparaison n'est pas raison. París: Gallimard.

- (1977). Ensayos de literatura (verdaderamente) general. Madrid: Taurus.

- (1988). Ouverture(s) sur un comparatisme planétaire. París: Christian Bourgois Editeur.

Even-Zohar I. (1990). «Polysystem Theory». Poetics Today 11: 1, 9-26.

FoKKEMA, D. (1996). «Comparative Literature and the Problem of Canon Formation». Canadian Review fo Comparative Literature XXIII 1, marzo, 51-66. Traducido al castellano en Romero López, D. (ed.) (1998a).

GILlesPIE, G. (1992). «Rhinoceros, Unicorn or Chimera? - A Poly-systemic View of Possible Kinds of Comparative Literature in the New Century». The Journal of Intercultural Studies 19, 14-21. Traducido al castellano en Romero López, D. (ed.) (1998a).

GONZÁLEZ DEL VALLE, L. (1993). El canon: reflexiones sobre la recepción literaria-teatral (Pérez de Ayala ante Benavente). Madrid: Huerga \& Fierro Editores.

GrAfF, G. (1987). Proffessing Literature: An Institucional History. Chicago: Chicago University Press .

- (1990). "Other Voices other Rooms: Organizing and Teaching the Humanities Conflict». New Literary History 21, 817-839.

GuILlÉN, C. (1971). Literature as System. Essays Toward the Theory of Literary History. Princeton: Princeton University Press.

- (1985). Entre lo uno y lo diverso. Barcelona: Crítica.

GuYARD, M.-F. (1951). La littérature comparée. París: Presses Universitaires de France.

KeRMODE, F. (1983). «Institutional Control of Interpretation». En The Art of Telling, Essays on Fiction. Cambridge: Harvard University Press.

- (1988). Formas de atención. Barcelona: Gedisa.

KUSHNER, E. (1995). «Towards a Typology of Comparative Literature Studies». En Proceedings of the XIIIth Congress of the International Comparative Literature Association. The Force of Vision. Powers of Narration. Literary Theory, G. Gillespie y otros (eds.), vol. 3, 502-510. Tokio: University of Tokio Press. Traducido al castellano en Romero López, D. (ed.) (1998a).

LEFEVERE, A. (1997). Traducción, reescritura y la manipulación del canon literario. Salamanca: El Colegio de España.

Mignolo, W. (1991). «Canons A(nd) Cross-Cultural Boundaries (Or, Whose Canon are we Talking about?)». Poetics Today 12: 1, 1-28.

Pozuelo Yvancos, J. M. ${ }^{a}$. (1995). El canon en la teoría literaria contemporánea. Valencia: Episteme. 
PRAWER, S. S. (1973). Comparative Literary Studies. Londres: Gerald Duckworth \& Co. Ltd.

REMAK, H. H. (1961). «Comparative Literature. Its definition and Function». En Comparative Literature. Method and Perspective, N. Stallknecht, $\mathrm{H}$. Frenz (eds.), 1-57. Carbondale \& Edwardsville: Southern Illinois University Press.

RiNnER, F. (1990). «Y a-t-il une théorie propre á la littérature comparée». En Actes du XIème congrès AILC: Toward a Theory of Comparative Literature, M. Valdés (ed.), vol. 3, 173-180. Berna: Peter Lang.

ROMERO LÓPEZ, D. (ed.) (1998a). Orientaciones en literatura comparada. Madrid: Arco Libros.

- (1998b). Una relectura del fin de siglo en el marco de la literatura comparada. Berna: Peter Lang.

Schmeling, M. (ed.) (1984). Teoría y praxis de la literatura comparada. Barcelona y Caracas: Alfa.

Schulz, H. \& Rhein, P. (eds.) (1973). Comparative Literature. The Early Years. North Carolina: The University of North Carolina Press.

SHEFFY, R. (1990). "The Concept of Canonicity in the PolysystemTheory». Poetics Today 11: 3, 53-61.

SMITH, B. H. (1983). «Contingencies of Value». Critical Inquiry 10, 1-35.

SteINer, G. (1992). Presencias reales. Barcelona: Lumen.

TALENS, J. (1994). «El lugar de la teoría de la literatura en la era del lenguaje electrónico». En Curso de teoría de la literatura, D. Villanueva (coord.), 129-143. Madrid: Taurus.

TöтÖsY, S. (1992). «Systemic Approaches to Literature. An Introduction with Selected Bibliographies». Canadian Review of Comparative Literature, marzo-junio, 21-93.

- (1998). Comparative Literature. Theory, Method, Application. Ámsterdam-Atlanta: Rodopi.

VAN TIEGHEM, P. (1931). La littérature comparée. París: Librairie Armand Colin.

Wellek, R. (1963). «The Crisis of Comparative Literature». En Concepts of Criticism, R. Wellek (ed.), 282-295. New Haven \& Londres: Yale University Press.

Wellek, R. y Warren, A. (1981). Teoría literaria. Madrid: Gredos. 